\title{
Hypoxia-inducible factor-1a polymorphisms link to coronary artery collateral development and clinical presentation of coronary artery disease
}

\author{
Qian Liu, Yun Liang, Ping Zou, Wei-xin Ni, Yu-guang Li, Song-ming Chen
}

\begin{abstract}
Aims. This study aimed to investigate the association of Hypoxia-inducible factor-1a (HIF-1a) C1772T and G1790A single nucleotide polymorphisms (SNPs) with: incidence, clinical type, severity of coronary atherosclerosis and coronary collaterals of coronary artery disease (CAD).

Methods. The clinical data and genomic DNA were gathered in 958 subjects, including 560 controls and 398 patients with CAD. CAD was confirmed with coronary angiography (CAG). The genotypes for two SNPs were determined by high resolution melting after PCR amplification.

Results. Neither the HIF-1 a C1772T nor the G1790A genotype was significantly associated with CAD and, no gene-gene or gene-environmental interactions were identified. However, both HIF-1 a C1772T and G1790A $(P<0.05)$ alleles were associated with clinical type and formation of coronary collaterals $(P<0.05)$. Patients carrying genotype $C T(P=0.019$, $\mathrm{OR}=4.905,91,95 \% \mathrm{Cl}: 1.355-17.761)$ and $\mathrm{GA}(P=0.026, \mathrm{OR}=3.052,95 \% \mathrm{Cl}: 1.180-7.892)$ had significantly higher stable angina pectoris (SAP) than unstable angina pectoris (UAP) and acute myocardial infarction (AMI). The presence of HIF-1 genotype $C T(P=0.016, \mathrm{OR}=13.373,95 \% \mathrm{Cl}$ : 15.468-32.709) and $\mathrm{GA}(P=0.001, \mathrm{OR}=19.741,95 \% \mathrm{Cl}: 8.125-47.966)$ predicted lower collateral formation and severity of $C A D$ secondary to the absence of collaterals $(r=0.242, P<0.001)$.

Conclusions. We conclude that functional polymorphisms in the HIF-1a gene do not modify CAD risk but they are associated with the formation of coronary collaterals and clinical presentation of CAD.
\end{abstract}

Key words: coronary artery disease, hypoxia-inducible factor, single nucleotide polymorphism, collaterals

Received: May 6, 2013; Accepted with revision: August 16, 2013; Available online: October 2, 2013

http://dx.doi.org/10.5507/bp.2013.061

Department of Cardiology, First Affiliated Hospital, Shantou University Medical College, Shantou, Guangdong 515041, China Corresponding author: Song-ming Chen, e-mail: cms1002@163.com

\section{INTRODUCTION}

Coronary artery disease (CAD) is a multifactorial heritable disease caused by a variety of risk factors ${ }^{1}$ relating to the oxygen and energy needs of myocardial tissue. Genetic polymorphisms are responsible for inter-individual variation and diversity and are considered the main genetic determinants of the development of some diseas$\mathrm{es}^{2}$. Production of hypoxia-inducible factor- $1 \alpha$ (HIF-1 $\left.\alpha\right)$ is a key regulator of cellular response to hypoxia ${ }^{3}$ and may participate in the occurrence and progression of CAD through activating various genes such as vascular endothelial cell growth factor (VEGF), hemeoxygenase-1 (HO-1), endothelin-1(ET-1) subsequently linking to regulation of angiogenesis, erythropoiesis, energy metabolism, vasomotor function and, apoptotic/proliferative responses ${ }^{4}$. In clinical observation, whether there is angiogenesis or not makes a great difference to the severity of chronic coronary ischemia. However, the formation of coronary collaterals varies between individuals.

$\mathrm{HIF}$ is a heterodimeric transcription factor consisting of $\alpha$ and $\beta$ subunits that sense the oxygen tension within the cell ${ }^{5}$. Under normoxic conditions, hydroxylation of Pro402 and Pro564 within the oxygen-dependent degradation domain (ODD) of the HIF-1 $\alpha$ protein leads to the binding of ODD with the von Hippel-Lindau tumor suppressor protein (pVHL), which functions as an E3 ubiquitin ligase predominantly targeting the minimal $\mathrm{N}$-terminal transactivation domain (N-TAD). Thereafter, the HIF-1 $\alpha$ protein is rapidly degraded through the pVHL-mediated ubiquitin-proteasome pathway ${ }^{6}$. Under hypoxic conditions, the hydroxylation of Pro402 and Pro564 decreases and HIF- $1 \alpha$ protein accumulates. HIF- $1 \alpha$ binds to HIF$1 \beta$, then translocates to the nuclear compartment and activates the transcription of hypoxia-inducible gene by binding to hypoxia response elements in promoters of a diverse variety of downstream target genes ${ }^{7-9}$.

Induction of HIF-1 $\alpha$ appears to be a crucial step in tissue response to hypoxia. Our previous study found that the HIF-1 $\alpha$ expression was significantly stronger in patients with CAD than controls, and the level of HIF-1 $\alpha$ was associated with severity of atherosclerosis and higher level of coronary collaterals ${ }^{10,11}$. Recently, two single nucleotide polymorphisms (SNPs) within the oxygen-dependent degradation domain of the HIF-1 $\alpha$ gene, HIF-1 C1772T (rs11549465) and G1790A (rs 11549467) were identified respectively ${ }^{12}$, and, feature with proline to serine (P582S) and alanine to threonine amino acid substitutions (A588T). These SNPs are thought to be important for protein stability as they locate within the ODD, very close to proline 564 (P564) and proline 402(P402) is a key for oxygen-dependent degradation of the HIF.

HIF-1 $\alpha$ SNPs relate to various types of diseases, such as acute myocardial infarction, type 1 and 2 diabetes mellitus and cancer. Mark and colleagues found that HIF $1 \alpha$ polymorphisms were associated with the development of 
stable exertional angina rather than AMI (ref. ${ }^{13}$ ). However, their study had no control group. Our study was designed to elucidate the association of HIF- $1 \alpha 1 \mathrm{C} 1772 \mathrm{~T}$ and G1790A polymorphisms with the incidence, clinical classification, extent of collateral formation and severity of CAD.

\section{MATERIALS AND METHODS}

\section{Subjects}

Subjects were enrolled from patients admitted to three hospitals (First Affiliated Hospital of Shantou University Medical College, Huaqiao Hospital in JieXi, and People's Hospital in Fengshun) in Guangdong Province, China, between 2007 to 2011. Patients were first suspected of CAD with clinical manifestations, certified with coronary angiography. Patients with the following conditions were exclusive: presence of severe related diseases such as anemia and tumors, prior revascularization by percutaneous coronary intervention or coronary artery bypass surgery, died or unsuccessful in coronary angiography imaging. 560 patients were finally included (case group). The control group comprised 398 healthly people over 18 years of age. All subjects were Chinese Hans, and all blood samples were collected with written informed consent from either the patients or their family members and with the approval of Shantou University Medical College Ethics Committee. The patients were further classified as 115 SAP (stable angina pectoris), 135 UAP(unstable angina pectoris), and 315 AMI. AMI was confirmed with ST-segment elevation and/or abnormal Q wave on an electrocardiogram, and elevated serum creatine kinase $(\mathrm{CK})$ levels. UAP and SAP were distinguished by having ischemic chest pain at rest or during positive activity, but neither provided any evidence of myocardial necrosis by enzymatic and electrocardiogram criteria. The characteristics of subjects including clinical variables as well as age, gender were summarized in Table 1.

Evaluation of coronary collateral formation was identified by two experimented angiographers under coronary angiography and Gensini score, illustrating how the severity of coronary artery was assessed as described previously $^{11}$. This method defines narrowing of the lumen of the coronary arteries as 1 score for $1-25 \%$ stenosis, 2 for $26-50 \%$, 4 for $51-75 \%, 8$ for $76-90 \%, 16$ for $91-99 \%$, and 32 for total occlusion. The score is then multiplied by a factor that represents the importance of the lesion's location in the coronary artery system. For the location scores, 5 points were given for the left main lesion; 2.5 for the proximal left anterior descending (LAD) or left circumflex (LCX) artery; 1.5 for the mid segment LAD and LCX; 1 for the distal segment of LAD and LCX, first diagonal branch, first obtuse marginal branch, right coronary artery, posterior descending artery, and intermediate artery; and 0.5 for the second diagonal and second obtuse marginal branches. Artery blood samples were obtained and heparin-anticoagulated as soon as artery puncture success DNA extraction and PCR. Genomic DNA was extracted from peripheral blood samples using a SiMax ${ }^{\mathrm{TM}}$ genomic DNA Kit (SBS Genetech Beijing China). Initially a small fragment containing the two SNP loci were amplified through the sequencing primer ( $r s F / r s R$ rsF5'GGATGATGACTTCCAGTTACG rsR 5' TGGTAGTGGTGGCATTAGC 3 '). Then HIF1 $\alpha$ polymorphisms were analysed by PCR amplification (rs 115 49465F:5'AGTTACGTTCCTTCGATCAGTTGTC3' rs 11549465R:5'CTTTCAGGGCTTGCGGAACTGCT TT3' rs 11549467F:5'CAGTTGTCACCATTAGAAAGC AGTT3' rs11549467R:5'TCTGCTGGAATACTGTAAC TGTGCT 3') and subsequent sequencing on the HRM instruments (LightScanner 96 system, Idaho company, USA). The accuracy of the genotyping was determined by sequencing using the reverse complementary strand (Invitrogen, USA).

\section{Statistics}

Statistical analysis was conducted using SPSS 17.0 software. Continuous variables were analyzed by the unpaired, two-sided t-test and presented as mean \pm standard deviation (SD) or $n(\%)$. Categorical variables are presented as counts or percentages and were compared by a Chi-square test or the Fisher exact test. Odds ratios (OR) and $95 \%$ confidence intervals (CI) were determined by logistic regression analysis. The Hardy-Weinberg equilibrium assumption was assessed using standard maximum likelihood methods. Single factor correlation analysis was performed using Spearman rank correlation analysis. The two degree of linkage disequilibrium between the sites and haplotype frequencies were tested using online software SHEsis (http://analysis.bio-X.cn/myAnalysis.php) (ref. ${ }^{14}$ ). MDR software was used to assess gene-gene and geneinvironment interaction. $P<0.05$ for the difference was considered statistically significant.

\section{RESULT}

The clinical characteristics of the participants are summarized in Table 1. Mean ages were $65.7 \pm 11.6$ years in cases and $59.8 \pm 13.8$ in controls $(P<0.001)$. An increased risk of $\mathrm{CAD}$ was associated with male sex, smoking, hypertension, diabetes and dyslipidemia. A significant difference in distribution of age, BMI, number of cigarettes smoked, systolic blood pressure (SBP), diastole blood pressure (DSP), glucose between CAD patients and controls was found.

Distribution of the HIF- $1 \alpha$ P582S and A588T allele and genotypes was not significant different in patients and controls (Table 2). Genotypes did not deviate from the Hardy-Weinberg equilibrium among groups and showed strong linkage disequilibrium ( $>>0.9$ ). Therefore, for further analysis we selected haplotypes constructed from these two SNPs (Table 1), whose frequency was at least 1\% (CG, CA and TG). No significant relationship was found between the haplotypes and incident rate of CAD.

Potential gene-environmental and gene-gene interactions on CAD risk were evaluated using MDR software. We found that four models were strongly predictive of 
Table 1. The distributions of clinical characteristics in controls and patients

\begin{tabular}{lcc}
\hline Characteristic & Control $\mathrm{n}=398$ & Case $\mathrm{n}=560$ \\
\hline Male** & $195(49.0)$ & $414(73.9)$ \\
Age** $^{*}$ & $59.8 \pm 13.8$ & $65.7 \pm 11.6$ \\
BMI $\left(\mathrm{kg} / \mathrm{m}^{2}\right)^{* *}$ & $22.1 \pm 6.2$ & $24.5 \pm 9.2$ \\
Family CAD** & $25(6.6)$ & $75(14.6)$ \\
Smoking** & $133(33.4)$ & $308(55.0)$ \\
Amount per day** & $6.5 \pm 10.8$ & $11.8 \pm 3.9$ \\
Years of smoking** & $10.6 \pm 16.7$ & $18.2 \pm 19.1$ \\
hypertension** & $178(44.7)$ & $322(57.5)$ \\
SBP (mmHg)* & $143.7 \pm 32.1$ & $149.3 \pm 30.9$ \\
DBP (mmHg)* & $85.2 \pm 17.6$ & $87.9 \pm 16.5$ \\
Diabetes mellitus** & $60(15.1)$ & $161(28.8)$ \\
Glucose (mm oi/U)** & $5.2 \pm 1.6$ & $7.8 \pm 4.6$ \\
Dyslipidemia history* & $74(18.6)$ & $144(25.7)$ \\
Cholesterol (mol/L) & $5.0 \pm 1.2$ & $5.1 \pm 1.3$ \\
high-density lipoprotein & $1.31 \pm 0.4$ & $1.30 \pm 0.5$ \\
low-density lipoprotein & $2.7 \pm 0.9$ & $2.8 \pm 0.9$ \\
triglycerides & $1.7 \pm 1.3$ & $1.9 \pm 1.1$ \\
\hline
\end{tabular}

data are expressed as mean $\pm \mathrm{SD}$ or $\mathrm{n}(\%) * * P<0.001 * P<0.05$

Table 2. HIF-1 $\alpha$ gene polymorphisms in CAD patients and controls.

\begin{tabular}{|c|c|c|c|c|c|}
\hline Gene & & Control & CAD & $P$ & OR(95\%CI) \\
\hline \multicolumn{6}{|l|}{$\mathrm{C} 1772 \mathrm{~T}$} \\
\hline \multirow[t]{2}{*}{ Allele (\%) } & $\mathrm{C}$ & $421(0.988)$ & $702(0.986)$ & 0.74 & $0.83 \quad(0.28-2.46)$ \\
\hline & $\mathrm{T}$ & $5(0.012)$ & $10(0.014)$ & & \\
\hline \multirow[t]{2}{*}{ Genotype (\%) } & $\mathrm{CC}$ & $208(0.977)$ & $346(0.972)$ & 0.74 & $0.831 \quad(0.28-2.47)$ \\
\hline & $\mathrm{CT}$ & $5(0.023)$ & $10(0.028)$ & & \\
\hline \multicolumn{6}{|l|}{ G1790A } \\
\hline \multirow[t]{2}{*}{ Allele (\%) } & $\mathrm{G}$ & 417 (0.979) & $696(0.978)$ & 0.88 & $0.94 \quad(0.41-2.14)$ \\
\hline & A & $9(0.021)$ & $16(0.022)$ & & \\
\hline \multirow[t]{2}{*}{ Genotype (\%) } & GG & $204(0.958)$ & $340(0.955)$ & 0.88 & $0.94 \quad(0.414-2.16)$ \\
\hline & GA & $9(0.042)$ & $16(0.045)$ & & \\
\hline \multicolumn{6}{|l|}{ C1772T-G1790A } \\
\hline \multirow[t]{3}{*}{ Haplotype (\%) } & CG & $412.00(0.967)$ & $686.00(0.963)$ & 0.75 & $0.897 \quad(0.46-1.74)$ \\
\hline & $\mathrm{CA}$ & $9.00(0.021)$ & $16.00(0.022)$ & 0.88 & $1.07 \quad(0.47-2.43)$ \\
\hline & TG & $5.00(0.012)$ & $10.00(0.014)$ & 0.74 & $1.20 \quad(0.41-3.53)$ \\
\hline
\end{tabular}

Table 3. MDR model of gene-environmental interactions.

\begin{tabular}{lcccc}
\hline Model & $\begin{array}{c}\text { Average Testing } \\
\text { Accuracy }\end{array}$ & $\begin{array}{c}\text { Cross Validation } \\
\text { Test }\end{array}$ & OR(95\%) pa & $P^{\mathrm{a}}$ \\
\hline X1 & 0.6378 & $10 / 10$ & $3.5095(2.3223,5.3036)$ & $<0.0001$ \\
X3 X5 & 0.6628 & $10 / 10$ & $4.6096(2.995,7.0933)$ & $<0.0001$ \\
X3 X5 X6 & 0.6781 & $10 / 10$ & $5.0057(3.3241,7.538)$ & $<0.0001$ \\
X1 X3 X5 X6 & 0.7091 & $10 / 10$ & $6.2984(4.1294,9.6065)$ & $<0.0001$ \\
\hline
\end{tabular}


Table 4. Association between HIF-1 $\alpha$ genotypes and clinical classification in patients.

\begin{tabular}{ccccccc}
\hline & CC $(\mathrm{n} \%)$ & CT $(\mathrm{n} \%)$ & $P$ & GG $(\mathrm{n} \%)$ & GA $(\mathrm{n} \%)$ & $P$ \\
\hline SAP & $61(92.4)$ & $5(7.6)$ & 0.029 & $59(89.4)$ & $7(10.6)$ & 0.044 \\
UAP & $59(98.3)$ & $1(1.7)$ & & $59(98.3)$ & $1(1.7)$ & \\
AMI & $196(98.5)$ & $3(1.5)$ & & $191(96)$ & $8(4)$ & \\
\hline
\end{tabular}

Table 5. Comparison of genotype distribution among SAP:UAP+AMI, UAP:SAP+AMI, and AMI: SAP+UAP.

\begin{tabular}{|c|c|c|c|c|c|}
\hline C1772T & CC (\%) & CT (\%) & $P$ & OR & $95 \% \mathrm{CI}$ \\
\hline SAP & $61(92.4)$ & $5(7.6)$ & & & \\
\hline $\mathrm{UAP}+\mathrm{AMI}$ & $255(98.5)$ & $4(1.5)$ & 0.019 & 4.905 & $1.355-17.761$ \\
\hline G1790A & GG (\%) & GA (\%) & $P$ & OR & $95 \% \mathrm{CI}$ \\
\hline SAP & $59(89.4)$ & 7 (10.6) & & & \\
\hline $\mathrm{UAP}+\mathrm{AMI}$ & $250(96.5)$ & $9(3.5)$ & 0026 & 3.052 & $1.180-7.892$ \\
\hline
\end{tabular}

Table 6. Association between HIF-1 $\alpha$ genotypes and collaterals in patients.

\begin{tabular}{lccccc}
\hline C1772T & CC & CT & $P$ & OR & $95 \%$ CI \\
No C ollaterals & 195 & 10 & & & 13.373 \\
Collaterals & 127 & 0 & 0.016 & OR & $95 \%$ CI \\
\hline G1790A & GG & GA & & & $8.468-32.709$ \\
No C ollaterals & 190 & 15 & 0.001 & 19.741 & 8.47 .966 \\
Collaterals & 127 & 0 &
\end{tabular}
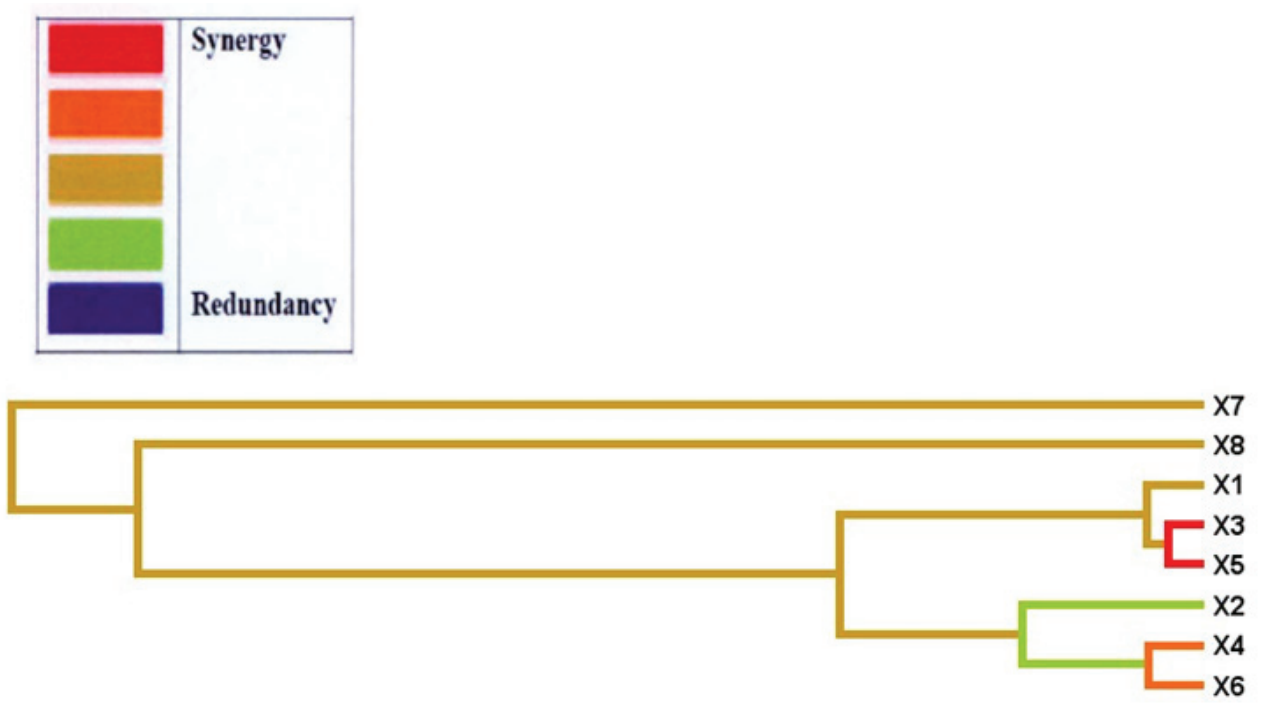

Fig. 1. The interaction between HIF polymorphism and environmental factors.

Colors from red to blue means the interaction of various becomes redundant. X1: Sex X2: Family history X3: Smoking history X4: History of hypertension X5: Diabetes history X6: Dyslipidemia history X7: C1772T X8: G1790A 
CAD $(\mathrm{P}<0.001$, cross-validation consistency $=10 / 10)$, but the accuracy of four-factor model was highest (average testing accuracy $=0.7001$ ), so the four-factor model is the best model. This contains sex, history of diabetes, dyslipidemia and smoking (Table 3). The interaction of smoking history and history of diabetes was strongest while the two SNPs in HIF gene interaction was weak (Fig. 1).

We examined the possible association of polymorphism and clinical classification in CAD and found both the HIF-1 $\alpha$ C1772T $(P<0.01)$ and G1790A $(P<0.05)$ allele was associated with clinical type (Table 4$)$. We further compared the genotypes distribution among SAP:UAP+AMI, UAP:SAP+AMI, and AMI :SAP+UAP, patients carrying genotype CT $(P=0.019 \mathrm{OR}=4.905,91$, 95\% CI: $1.355-17.761)$ and $\mathrm{GA}(P=0.026, \mathrm{OR}=3.052,95 \%$ CI: 1.180-7.892) was significant higher in SAP (Table 5). However, the genotype was not related with severity of CAD ( $P>0.05)$.

Among patients with collaterals, the frequency of the CT and GA genotype was 0.49 and 0.73 , respectively. Patients with both CT and GA genotype showed no collateral formation (Table 6). The frequency of the CT and GA genotype was significantly higher among patients without collaterals compared to patients with collaterals $(P<0.05)$. The absence of collaterals was significantly increased in patients with CT $(P=0.016, \mathrm{OR}=13.373,95 \%$ CI: $15.468-32.709)$ and GA $(P=0.001, O R=19.741,95 \%$ CI: 8.125-47.966) genotype compared with $\mathrm{CC}$ and GG genotype. However, the genotype was not related with severity of coronary artery disease $(P>0.05)$.

\section{DISCUSSION}

CAD is characterized by both familial aggregation and obvious individual differences. Understanding the inherited factors that influence susceptibility for developing various diseases may lead to achieving better and more comprehensive therapies ${ }^{15}$. In our study, we found that CAD patients with the C1772T and/or G1790A polymorphic variants had significant higher SAP and less collateral formation than those without the variant allele. However, we found no relation between polymorphisms of HIF- $1 \alpha$ and susceptibility to CAD.

HIF- $1 \alpha$ triggers VEGF expression which increase plaque capillary density and extends the depth of neovascularization to the intima ${ }^{16}$, resulting in mural hemorrhage, plaque rupture, and induces acute coronary syndrome (ACS) ( ref. $^{17}$ ). We conclude that the mutation of HIF gene may weaken the ability of HIF-1 $\alpha$ transcriptional activation ability to affect the expression of VEGF result in reducing collateral formation and decreaseing the incidence of ACS. In vitro data have shown that the HIF-1 $\alpha$ C1772T and G1790A polymorphisms have lower transcriptional activity than wild-type alleles at comparable expression levels ${ }^{18}$. Our findings also confirm that HIF1a polymorphisms link to clinical classification. Coronary collateral vessels can increase blood supply in arteries with high-grade stenosis, thus protecting the myocardium from ischemia ${ }^{19}$ This seems to conflict with our finding that polymorphism decreases collateral circulation and at the same time decrease the incidence of ACS. We hypothesize in the setting of an acute coronary occlusion, coronary collateral vessels would be unlikely to develop rapidly enough to protect against an AMI (ref. ${ }^{18}$ ). Alternatively, mutation may lead to reduced expression of glucose transporters and glycolytic enzymes, resulting in reduced metabolic adaptation to ischemia and earlier clinical presentation prior to extensive collateral formation $^{15}$.

To date, there have been few reports on the relationship between HIF-1 $\alpha$ polymorphisms and the incidence rate of CAD. This is in conflict with our first hypothesized. In other words, environmental factors play a leading role and not gene factors in the pathogenesis of CAD. There are some possible explanations, firstly, the statistical power was low owing to the relatively small sample size and the low frequency of variant alleles, thus, the associations observed between HIF-1 $\alpha$ C1772T, G1790A and CAD risk may change; secondly, the number of candidate genes and mutations were limited, and could not exclude other minor interactions between them; and lastly, we presume the possibility that allelic variants did not regulate the expression of hypoxia-inducible factor $1 \alpha$ $\left(\right.$ ref. $\left.^{20-22}\right)$.

It seems a confusing result that genotype is related to the clinical manifestations of CAD but there is no significant correlation with severity of coronary artery lesion. We believe that the clinical manifestations of CAD do not reflect the extent of coronary artery lesion. These are due to multiple factors. For this reason, we should fully take into account environmental factors, such as patient age, gender and family history together with blood pressure, blood lipids and blood glucose. Moreover, although coronary angiography has been considered the gold standard for diagnosing CAD for a long time, there are limitations to evaluation of the characteristics of the plaque and the artery wall firstly, angiography can only reflect the lumen contours filled in by contrast agent. This means when the extent of coronary artery stenosis is less than $40 \%$, it cannot be found by coronary angiography, and coronary angiography cannot show coronary blood flow in the myocardium either. Secondly, the coronary lesions are often misshapen in the lumen or irregular and the limitations of projection imaging will affect the assessment of the degree of vascular stenosis, thirdly, coronary atherosclerosis is often diffuse, and so-called "normal" reference vessel segments may not be the normal standard. In this case, it will underestimate the severity of coronary angiography. Further, in the early stages of CAD, vascular lesions can occur by compensatory expansion. In this case coronary angiography may also underestimate the severity. Another possibility is that severe coronary artery lesions activate compensatory mechanisms to protect the heart, so that serious clinical manifestations may not occur. 


\section{CONCLUSIONS}

HIF-1 gene polymorphisms C1772T and G1790A are not correlated with incidence of CAD or degree of stenosis of coronary artery but they do associate with clinical type and collateral formation, which suggest that polymorphisms in candidate genes may provide prognostic information on cardiac risk factors.

\section{ACKNOWLEDGEMENT}

This work was supported by a Natural Scientific Fund Project of Guangdong Province, China (No. 07008215) and Guangdong Province Program for Tackling Key Scientific and Technology Program Problems (No 2008B030301241).

\section{CONFLICT OF INTEREST STATEMENT}

Author's conflict of interest disclosure: None declared.

\section{REFERENCES}

1. Milewicz DM, Seidman CE. Genetics of cardiovascular disease. Circulation 2000; 102(20 Suppl 4): IV103-11.

2. Rannala B. Finding genes influencing susceptibility to complex diseases in the post-genome era. Am J Pharmacogenomics 2001;1(3):203-21.

3. Smaldone MC, Maranchie JK. Clinical implications of hypoxia induc ible factor in renal cell carcinoma. Urol Oncol 2009;27(3):238-45.

4. Adams JM, Difazio LT, Rolandelli RH, Lujan JJ, Hasko G, Csoka B, et al. HIF-1: a key mediator in hypoxia. Acta Physiol Hung 2009;96(1):1928.

5. Dery MA, Michaud MD, Richard DE. Hypoxia-inducible factor 1: regulation by hypoxic and non-hypoxic activators. Int J Biochem Cell Biol 2005;37(3):535-40.

6. Kim HO, Jo YH, Lee J, Lee SS, Yoon KS. The C1772T genetic polymorphism in human HIF-1 alpha gene associates with expression of HIF-1alpha protein in breast cancer. Oncol Rep 2008;20(5):1181-7.

7. Wilhide $M E$, Jones WK. Potential therapeutic gene for the treatment of ischemic disease: Ad2/hypoxia-inducible factor-1alpha (HIF-1)/ VP16 enhances B-type natriuretic peptide gene expression via a HIF-1-responsive element. Mol Pharmacol 2006;69(6):1773-8.
8. Li YG, Wang DM, Chen SM, Tan XR, Fang XY, Wu JW. Haem oxygenase-1 expression and coronary heart disease--association between levels of haem oxygenase-1 expression and angiographic morphology as well as the quantity of coronary lesions. Acta Cardiol 2006;61(3):295-300

9. Fransen K, Fenech M, Fredrikson M, Dabrosin C, Soderkvist P. Association between ulcerative growth and hypoxia inducible factor-1alpha polymorphisms in colorectal cancer patients. Mol Carcinog 2006;45(11):833-40.

10. Chen SM, Li YG, Wang DM, Zhang GH, Tan CJ. Expression of heme oxygenase-1, hypoxia inducible factor-1alpha, and ubiquitin in peripheral inflammatory cells from patients with coronary heart disease. Clin Chem Lab Med 2009;47(3):327-33. doi: 10.1515/CCLM.2009.073

11. Chen SM, Li YG, Zhang HX, Zhang GH, Long JR, Tan CJ. Hypoxiainducible factor-1alpha induces the coronary collaterals for coronary artery disease. Coron Artery Dis 2008;19(3):173-9.

12. Zhao T, Lv J, Zhao J, Nzekebaloudou M. Hypoxia-inducible factor1alpha gene polymorphisms and cancer risk: a meta-analysis. J Exp Clin Cancer Res 2009;28:159.

13. Hlatky MA, Quertermous T, Boothroyd DB, Priest JR, Glassford AJ, Myers RM. Polymorphisms in hypoxia inducible factor 1 and the initial clinical presentation of coronary disease. Am Heart J 2007;154(6):1035-42.

14. Shi YY, He L. SHEsis, a powerful software platform for analyses of linkage disequilibrium, haplotype construction, and genetic association at polymorphism loci. Cell Res 2005;15(2):97-8.

15. Exner M, Minar E, Wagner O, Schillinger M. The role of heme oxygenase-1 promoter polymorphisms in human disease. Free Radic Biol Med 2004;37(8):1097-104.

16. Celletti FL, Hilfiker PR, Ghafouri P, Dake MD. Effect of human recombinant vascular endothelial growth factor165 on progression of atherosclerotic plaque. J Am Coll Cardiol 2001;37(8):2126-30.

17. Shah PK. Pathophysiology of plaque rupture and the concept of plaque stabilization. Cardiol Clin 2003;21(3):303-14

18. Resar JR, Roguin A, Voner J, Nasir K, Hennebry TA, Miller JM. Hypoxiainducible factor 1alpha polymorphism and coronary collaterals in patients with ischemic heart disease. Chest 2005;128(2):787-91.

19. Koerselman J, van der GraafY, de Jaegere PP, Grobbee DE. Coronary collaterals: an important and underexposed aspect of coronary artery disease. Circulation 2003;107(19):2507-11.

20. Nagy G, Kovacs-Nagy R, Kereszturi E, Somogyi A, Szekely A, Nemeth $\mathrm{N}$. Association of hypoxia inducible factor- 1 alpha gene polymorphism with both type 1 and type 2 diabetes in a Caucasian (Hungarian) sample. BMC Med Genet 2009;10:79.

21. Foley R, Marignol L, Thomas AZ, Cullen IM, Perry AS, Tewari P. The HIF-1alpha C1772T polymorphism may be associated with susceptibility to clinically localised prostate cancer but not with elevated expression of hypoxic biomarkers. Cancer Biol Ther 2009;8(2):118-24.

22. Percy MJ, Mooney SM, McMullin MF, Flores A, Lappin TR, Lee FS. A common polymorphism in the oxygen-dependent degradation (ODD) domain of hypoxia inducible factor-1alpha (HIF-1alpha) does not impair Pro-564 hydroxylation. Mol Cancer 2003;2:31. 